\title{
ON CONVERGENCE THEOREMS FOR NONABSOLUTE INTEGRALS
}

\author{
Lee Peng Yee and Chew tuan Seng
}

In this paper the Perron integrability of the limit of a sequence of functions is proved under certain convergence conditions.

\section{Introduction and the Perron integral}

The Denjoy, Perron and Henstock-Kurzweil integrals are known to be equivalent, $[4,5,8]$. They are nonabsolute in the sense that if a function $f$ is integrable, then its absolute value $|f|$ may or may not be integrable. Note that the Lebesgue integral is absolute and is included in any of the above integrals. Recently, some convergence theorems, which are in some sense better than the dominated convergence theorem, have been proved for the Denjoy and Henstock-Kurzweil integrals $[2,3,6,7]$. In this note, we shall prove two convergence theorems for the Perron integral, which are generalizations of the well-known dominated convergence theorem and the mean convergence theorem respectively.

A function $H$ is said to be a Perron major function of a function $f$ in $[a, b]$ if

$$
-\infty \neq \underline{D} H(x) \geq f(x) \text { for every } x
$$

where $\underline{D}$ denotes the lower derivative. A function $G$ is said to be a Perron minor function of $f$ in $[a, b]$ if $-G$ is a Perron major function of $-f$ in $[a, b]$. A function $f$ is said to be Perron integrable on $[a, b]$ if

$$
\inf \{H(b)-H(a)\}=\sup \{G(b)-G(a)\} \neq \pm \infty
$$

Received 11 October 1985. The authors are grateful to Professor Bullen for suggesting the use of the Marcinkiewicz theorem in the proof of the lemma and to Professor vyborny for an interesting discussion which led to the formulation of Theorem 4 and its proof.

Copyright Clearance Centre, Inc. Serial-fee code: 0004-9727/86 $\$ A 2.00+0.00$. 
where the infimum is over all Perron major functions $H$ of $f$ in $[a, b]$ and the supremum over all Perron minor functions $G$ of $f$ in $[a, b]$. The common value is defined to be the Perron integral of $f$ on $[a, b]$. For brevity, given a function $F$ we shall write $F(u, v)=F(v)-F(u)$.

We shall consider the following three conditions:

(i) $f_{n}(x) \rightarrow f(x)$ almost everywhere in $[a, b]$ as $n \rightarrow \infty$ where each $f_{n}$ is Perron integrable on $[a, b]$;

(ii) the primitives $F_{n}$ of $f_{n}$ converge uniformly on $[a, b]$;

(iii) $f_{n}$ have at least one common major function and at least one common minor function in $[a, b]$.

The idea of common major and minor functions is useful. For example, we can easily prove the following result.

THEOREM 1. If $f$ and $g$ are Perron integrabie on $[a, b]$ and have at least one common continuous major finction and at least one common continuous minor function in $[a, b]$, then $\max (f, g)$ and $\min (f, g)$ are also Perron integrable on $[a, b]$.

In fact, the above result is a special consequence of Marcinkiewicz's theorem [8; p. 253] and it remains valid for a family of functions instead of just two functions $f$ and $g$.

Now we state our first main result whose proof is given in section 2 . The second main result is Theorem 4 to be given in section 3 .

THEOREM 2. If conditions (i), (ii) and (iii) hold, then $f$ is Perron integrable on $[a, b]$ and we have

$$
\int_{a}^{b} f_{n}(x) d x \rightarrow \int_{a}^{b} f(x) d x \text { as } n \rightarrow \infty \text {. }
$$

In view of the remark after Theorem 1 , we see immediately that if both common major and minor functions are continuous then $f_{n}$ is dominated by inf $f_{n}$ and sup $f_{n}$, both Perron integrable. Thus the above theorem reduces to the dominated convergence theorem.

In general, the common major and minor functions are not continuous, as shown in the following example. Let

$$
\begin{aligned}
F(x) & =x^{2} \sin \left(1 / x^{2}\right) & \text { when } 0<x \leq 1, \\
& =0 & \text { when } x=0 .
\end{aligned}
$$


Define $f_{n}(x)=F^{\prime}(x)$ when $1 / n \leq x \leq 1$ and $f_{n}(x)=0$ otherwise. Then $f_{n}$ satisfies conditions (i), (ii) and (iii). Note that the common major function is given by $H(x)=H_{1}(x)+H_{2}(x)$ where $H_{1}(x)=F(x)$ when $F$ is increasing and 0 otherwise and $H_{2}(x)$ is a continuous and increasing function such that $H_{2}(a)=0, H_{2}(b)<\varepsilon$, and $H_{2}^{\prime}(x)=+\infty$ for the points $x$ at which $H_{1}(x)$ has a jump. Burkill [1] shows that such $H_{2}$ exists. The common minor function can be defined similarly.

\section{Controlled convergence}

We shall define the controlled convergence of a sequence of functions. First a function $F$ is said to be absolutely continuous in the restricted sense on $X$ or, in short, $A C_{*}(X)$, if for every $\varepsilon>0$ there is an $n>0$ such that for every finite or infinite sequence of non-overlapping intervals $\left\{\left[a_{i}, b_{i}\right]\right\}$ with $a_{i} b_{i} \in X$ and $\left\{\sum_{i}\left|b_{i}-a_{i}\right|<n\right.$ we have

$$
\sum_{i} w\left(F ;\left[a_{i}, b_{i}\right]\right)<\varepsilon
$$

where $\omega$ denotes the oscillation of $F$ over $\left[a_{i}, b_{i}\right]$. Further $F$ is said to be generalized absolutely continuous in the restricted sense on $[a, b]$ or $A C G_{*}$ if $[a, b]$ is the union of a sequence of closed sets $X_{i}$ such that on each $X_{i}$ the function $F$ is $A C_{*}\left(X_{i}\right)$. Similarly, we can define $V B_{*}(X)$ where $V B$ stands for bounded variation (see [8]).

In addition to (i) - (ii), we need the following conditions:

(iv) the primitives $F_{n}$ of $f_{n}$ are $A C G_{*}$ uniformly in $n$, that is, $[a, b]$ is the union of a sequence of closed sets $X_{i}$ such that on each $X_{i}$ the functions $F_{n}$ are $A C_{*}\left(X_{i}\right)$ uniformly in $n ;$ in other words, $n>0$ in the definition of $A C_{*}\left(X_{i}\right)$ is independent of $n$;

(v) $[a, b]$ is the union of a sequence of closed sets $X_{i}$ and for every $i$ and $\varepsilon>0$ there is an integer $N$ such that for every partial division of $[a, b]$ given by

$$
a \leq a_{1}<b_{1} \leq a_{2}<b_{2} \leq \ldots \leq a_{p}<b_{p}<b
$$


with $a_{1}, b_{1}, a_{2}, b_{2}, \ldots, a_{p}, b_{p} \in X_{i}$ we have

$$
\left|\sum_{j=1}^{p}\left\{F_{n}\left(a_{j}, b_{j}\right)-F_{m}\left(a_{j}, b_{j}\right)\right\}\right|<\varepsilon
$$

whenever $n, m \geq N$.

A sequence of functions $f_{n}$ is said to be control-convergent to $f$ if conditions (i), (ii) and (iv) are satisfied. The following lemma gives sufficient or necessary conditions for controlled convergence.

LEMMA. Suppose that (i) and (ii) hold. Then (iii) implies (iv), and (iv) implies (v).

Proof. Suppose that (iii) holds. Following the second half of the proof of Marcinkiewicz's theorem, [8; p. 253], we obtain that every closed subset $P$ of $[a, b]$ contains a portion $Q$ on which each $f_{n}$ is Lebesgue integrable, and that $f_{n}$ have a common major function and a common minor function which are both $V B_{*}(Q)$. Furthermore, let $I$ be the smallest interval containing $Q$. Then

$$
\sum_{i} \omega\left(F_{n} ;\left[a_{i}, b_{i}\right]\right)
$$

converges uniformly in $n$ for the sequence of the intervals $\left[a_{i}, b_{i}\right]$ in $I$ contiguous to $Q$. In view of ( $i i)$, the above series of oscillations is also convergent uniformly in $n$ for the sequence of the intervals $\left[a_{i}, b_{i}\right]$ in $[a, b]$ contiguous to $Q$.

Next, we observe that continuous functions $F_{n}$ are $A C G_{*}$ uniformly in $n$ if and only if every closed subset of $[a, b]$ contains a portion on which the functions $F_{n}$ are $A C_{*}$ uniformly in $n,[8 ;$ p. 233, Theorem 9.1]. Therefore we have (iv).

It is known, [6; Lemma 4], that (i), (ii) and (iv) imply (v).

In view of the lemma, Theorem 2 follows from the following controlled convergence theorem which was originally proved for the Henstock-Kurzweil integral, [6].

THEOREM 3. If $f_{n}$ is control-convergent to $f$, then $f$ is Perron integrable on $[a, b]$ and we have 


$$
\int_{a}^{b} f_{n}(x) d x \rightarrow \int_{a}^{b} f(x) d x \text { as } n \rightarrow \infty
$$

We remark (see [7]) that condition (ii) in the definition of the controlled convergence can be replaced by

(vi) $E_{n}$ are equicontinuous in $[a, b]$.

The above theorem with (ii) replaced by (vi) was proved by Džvaršeíšvili using the Dejoy integral, [2; p. 50 Theorem 47] or [3].

We further remark that a direct proof of Theorem 2 using the same argument as Marcinkiewicz's theorem is also possible.

\section{Generalized mean convergence}

Let $f_{n}$ be Perron integrable on $[a, b]$ with primitive $F_{n}$. Then $f_{n}$ is said to be generalized mean convergent on $[a, b]$ if (ii) and the following condition hold:

(vii) $[a, b]$ is the union of a sequence of closed sets $X_{i}$ and for every $i$ and $\varepsilon>0$ there is an integer $N$ such that for every partial division of $[a, b]$ given as in (v) we have

$$
\sum_{j=1}^{p} \omega\left(F_{n}-F_{m} ;\left[a_{j}, b_{j}\right]\right)<\varepsilon
$$

whenever $n, m \geq N$.

We remark that when $X_{i}=[a, b]$ for all $i$, both conditions $(v)$ and (vii) are equivalent to the mean convergence of $f_{n}$ on $[a, b]$, hence the term generalized mean convergence above.

THEOREM 4. Let $f_{n}$ be Perron integrable on $[a, b]$ and generatized mean convergent on $[a, b]$. Then there exists a function $f$ which is Perron integrable on $[a, b]$ and there exists a subsequence $f_{n(i)}$ of $f_{n}$ such that $f_{n(i)}(x) \rightarrow f(x)$ almost everywhere in $[a, b]$ as $n(i) \rightarrow \infty$ and that $f_{n(i)}$ is generalized mean convergent to $f$ on $[a, b]$. Furthermore,

$$
\int_{a}^{b} f_{n}(x) d x \rightarrow \int_{a}^{b} f(x) d x \text { as } n \rightarrow \infty
$$

Proof. Let conditions (ii) and (vii) be satisfied. Fix $X_{1}$ and let 
$\left[a_{k}, b_{k}\right], k=1,2, \ldots$ be the sub-intervals of $[a, b]$ contiguous to $X_{1}$. We may assume $a, b \in X_{1}$. Consider the sequence $F_{n}$ and define $G_{n}(x)=F_{n}(x)$ when $x \in X_{1}$ and piecewise linear on the complement of $X_{1}$ such that

$$
\omega\left(G_{n} ;\left[a_{k}, b_{k}\right]\right)=\omega\left(F_{n} ;\left[a_{k}, b_{k}\right]\right) \text { for } k=1,2, \ldots
$$

Let $g_{n}(x)=G_{n}^{\prime}(x)$ almost everywhere. Then $g_{n}$ is mean convergent on $[a, b]$, that is

$$
\int_{a}^{b}\left|g_{n}(x)-g_{m}(x)\right| d x \rightarrow 0 \text { as } n, m \rightarrow \infty
$$

Hence there is a subsequence $g_{n(i)}$ of $g_{n}$ which is dominated on the right by $g_{n(1)}+h$ and on the left by $g_{n(1)}-h$ where

$$
h=\sum_{i=1}^{\infty}\left|g_{n(i)}-g_{n(i+1)}\right| .
$$

Furthermore, $g_{n(i)}(x)$ converges almost everywhere to a Perron integrable function $g$ on $[a, b]$. Note that $g_{n(i)}(x)=f_{n(i)}(x)$ almost everywhere in $X_{1}$. Hence $f_{n(i)}(x)$ converges almost everywhere in $X_{1}$ to a function $f(x)$ where $f(x)=g(x)$ when $x \in X_{1}$.

Since $f_{n(1)}$ is Perron integrable on $[a, b]$ and its primitive $E_{n(1)}$ is $A C G_{*}$, then $[a, b]$ is the union of closed sets $y_{j}, j=1,2, \ldots$ such that $F_{n(1)}$ is $A C_{*}\left(Y_{j}\right)$ for each $j$. In view of $g_{n(i)}$ being dominated by $g_{n(1)}+h$ and $g_{n(1)}-h$, we see that the functions $G_{n(i)}$ and, therefore, $F_{n(i)}$, are $A C_{*}\left(X_{1} \cap Y_{j}\right)$ uniformly in $n(i)$ for each $j$.

Repeat the above process for $X_{2}$ and the sequence $F_{n(i)}$ obtained above and so on. Consequently, by the diagonal process, we obtain a new subsequence $f_{n(i)}$ of $f_{n}$ such that $f_{n(i)}(x) \rightarrow f(x)$ almost everywhere in $[a, b]$ as $n(i) \rightarrow \infty$ and that $f_{n(i)}$ is generalized mean convergent to $f$ on $[a, b]$ with primitives $F_{n(i)}$ being $A C G_{*}$ uniformly in $n(i)$. Then it follows from Theorem 3 that $f$ is Perron integrable on $[a, b]$ and we have 


$$
\int_{a}^{b} f_{n(i)}(x) d x \rightarrow \int_{a}^{b} f(x) d x \text { as } n(i) \rightarrow \infty
$$

Since every subsequence of $f_{n}$ has a sub-subsequence which satisfies the above expression, it holds for the sequence $f_{n}$ itself, and the proof is complete.

It follows from the proof above that if $f_{n}$ is generalized mean convergent on $[a, b]$, then a subsequence of $f_{n}$ is control-convergent to some function $f$ on $[a, b]$. Similarly, we can prove that if $f_{n}$ is control-convergent to $f$ on $[a, b]$, then there is a subsequence of $f_{n}$ satisfying (iii). We remark that a simple and direct proof of Theorem 4 using the Henstock-Kurzweil integral is also possible (see [5; p. 86]).

The equivalence theorem in [7] states that $f$ is Perron integrable on $[a, b]$ if and only if there is a control-convergent sequence $\left\{\phi_{n}\right\}$ of step functions such that $\phi_{n}(x) \rightarrow f(x)$ almost everywhere in $[a, b]$ as $n \rightarrow \infty$. Consequently, Theorem 4, together with the equivalence theorem in [7], gives the following alternative definition of the perron integral.

THEOREM 5. A function $f$ is Perron integrable on $[a b]$ if and only if there is a sequence of step functions which is generalized mean convergent to $f$ on $[a, b]$.

From a functional analytic point of view, the space of Perron integrable functions is nothing but the completion of the class of all step functions under generalized mean convergence. As we can see, Theorem 2 is a natural extension of the dominated convergence theorem and Theorem 4 that of the mean convergence theorem. Since the Denjoy, Perron and HenstockKurzweil integrals are all equivalent, the various convergence theorems are valid for all three integrals.

\section{References}

[1] J.C. Burkill, "The approximately continuous Perron integral", Math. Z. 34 (1931), 270-278.

[2] V.G. Celidze and A.G. Džvaršeísvili, Theory of the Denjoy integral and some of its applications, in Russian, (Tbilisi, 1978). 
[3] A.G. Džvarseǐsvili, "On a sequence of integrals in the sense of Denjoy", Akad. Nauk Gmuzin. SSR. Trudy Mat. Inst. Rajmadze 18 (1951), 221-236.

[4] R. Henstock, Theory of integration, (London, 1963).

[5] R. Henstock, "A Riemann-type integral of Lebesgue power", Canad. $J$. Math. 20 (1968), 79-87.

[6] Lee Peng Yee and Chew Tuan Seng, "A better convergence theorem for Henstock integrals", BulZ. London Math. Soc. 17 (1985) 557-564.

[7] Lee Peng Yee and Chew Tuan Seng, "A Riesz-type definition of the Denjoy integral", Real Anal. Exchange 11 (1985/86) No.1, 221-227.

[8] S. Saks, Theory of the integral, 2nd ed., (Warsaw, 1937).

National University of Singapore

Republic of Singapore 